\title{
Emissive Exciplexes of Surface-Immobilized Dybenzoylmethanatoboron Difluoride with Gaseous Benzene, Toluene and Xylenes
}

\author{
Mikhail Alfimov ${ }^{1}$, Viacheslav Sazhnikov ${ }^{1}$, Aziz Muzafarov ${ }^{2}$ \\ ${ }^{1}$ Photochemistry Center of RAS, Novatorov St. 7a-1, 119421, Moscow, Russia \\ alfimov@photonics.ru \\ ${ }^{2}$ Enikolopov Institute of Synthetic Polymer Materials of RAS , Profsoyuznaya St. 70, 117393, \\ Moscow, Russia
}

\begin{abstract}
:
Core-shell silica nanoparticles consisting of a $\mathrm{SiO}_{2}$ core and a shell with covalently attached alkoxysilyl derivative of dibenzoylmethanatoboron difluoride $\left(\mathrm{DBMBF}_{2}\right)$ were synthesized. It was found that the nanoparticles may be used as the basis of selective sensor materials suitable for detecting benzene, toluene and xylene vapors. The $\mathrm{DBMBF}_{2}$ fluorescence quenching followed by the appearance of the $\mathrm{DBMBF}_{2}$ /analyte exciplex fluorescence is observed upon adsorption of benzene or its methyl derivatives on the nanoparticle surface. The position of the isoemissive point is characteristic of each analyte and can be used for its identification.
\end{abstract}

Key words: BTX, exciplex, fluorescence, nanoparticle, isoemissive point.

\section{Introduction}

Benzene, toluene and xylenes (BTX) are VOCs of great environmental significance. But the greatest health risk from exposure to BTX is known to be due to benzene. The OSHA's permissible exposure levels for benzene is 1 $\mathrm{ppm}$ as compared to $100 \mathrm{ppm}$ for toluene and xylenes. Therefore, the development of materials exhibiting both high sensitivity and good selectivity towards the individual BTX components is one of the most interesting and difficult challenges.

It is known that formation of exciplexes between dibenzoylmethanatoboron difluoride $\left(\mathrm{DBMBF}_{2}\right)$ and benzene or methyl-substituted benzenes in solutions is one of the most characteristic properties of the fluorophore [1-3]. The fluorescence characteristics of these exciplexes depend on the methylbenzene nature. In principle, this fact can be used for the development of new selective sensor materials. Recently, it was shown that $\mathrm{DBMBF}_{2}$ immobilized on silica gel can also form fluorescent exciplexes with toluene and $\mathrm{m}$ xylene adsorbed from the vapor phase $[4,5]$. In this work, an alkoxysilyl derivative of $\mathrm{DBMBF}_{2}$ was synthesized and then covalently grafted onto the surface of synthesized silica nanoparticles (SNP). The goal was to study the spectral features of exciplexes formed between
$\mathrm{DBMBF}_{2}$ covalently linked to the nanoparticle surface and vapor-phase adsorbed benzene, toluene and xylenes.

\section{Experimental}

${ }^{1} \mathrm{H}$ and ${ }^{13} \mathrm{C}$ NMR and ${ }^{19} \mathrm{~F}$ NMR spectra were measured on a Bruker Avance II 300 spectrometer. IR spectra were recorded with a Bruker Equinox 55/S spectrometer. TEM photographs were taken on a Leo $912 \mathrm{AB}$ Omega (Germany) transmission electron microscope. Fluorescence responses to the vapour exposure were obtained in a closed box (volume 1.7 L) where a definite pressure of BTX vapors were created by evaporating a certain amount of an alkylbenzene $(0.05-015 \mathrm{~mL})$. A small electrical fun was used to obtain uniform vapour distribution over the box volume. The samples were prepared by drop-casting a SNP dispersion onto the surface of a TLC plate (Sorbfil, silica gel sorbent layer of 110-120 $\mu \mathrm{m}$ thickness, sorbent size 5-17 $\mu \mathrm{m})$. Changes in the fluorescence spectra were registered using an R400-7 UV/VIS fiber-optic fluorescent probe inserted into the box and attached to a D-2000 Ocean Optic fiber-optic spectrofluorimeter. A LED $(375 \mathrm{~nm})$ was used as a light source. The fluorescence responses of the samples at low analyte concentrations (10-50 ppm) were studied using a special experimental system designed for this purpose [6]. 


\section{Results and Discussion}

Monodisperse silica nanoparticles with diameters ranging from 90 to $100 \mathrm{~nm}$ were obtained by hydrolytic polycondensation of tetraethoxysilane in mixtures of ethanol, water and ammonia. The synthesis of alkoxysilyl derivative of $\mathrm{DBMBF}_{2}$ involved several stages shown in Fig.1.

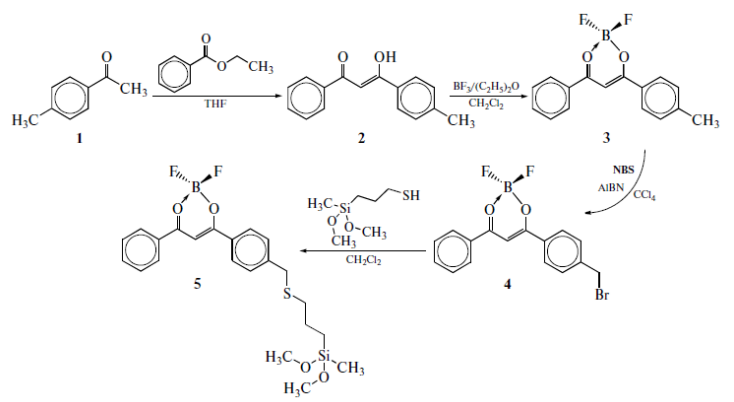

Fig. 1. Schema of four-step synthesis of an alkoxysylil derivative of $D B M B F_{2}$.

Schematic representation of the synthesis of $\mathrm{DBMBF}_{2}$ grafted SNP is given in Fig. 2.

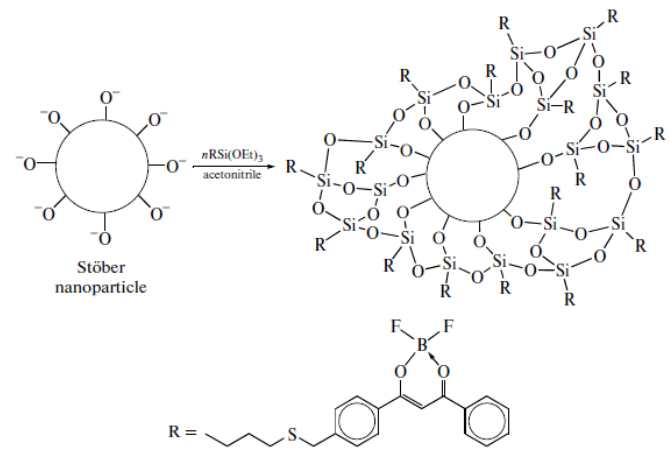

Fig. 2. Schematic of the synthesis of silica nanoparticles with covalently linked $D B M B F_{2}$.

The TEM microphotograps show that the shell thickness is about 2-4 $\mathrm{nm}$.

Figs. 3-6 show the time evolution of the fluorescence emission spectra of TLC-plate samples containing dispersed $\mathrm{SiO}_{2}$ nanoparticles with covalently grafted $\mathrm{DBMBF}_{2}$ during exposure to saturated BTX vapors (frontface configuration, $\lambda_{\mathrm{ex}}=375 \mathrm{~nm}$, room temperature). The values of the fluorescence maxima and isoemissive points are shown. Spectra were obtained at every $4 \mathrm{~s}$ from the beginning of exposure to vapors. Surfaceimmobilized $\mathrm{DBMBF}_{2}$ exhibits a single fluorescence band centered at $425 \mathrm{~nm}$. Upon exposure to BTX vapors, the intensity of this band is decreased (except benzene) and broad red-shifted fluorescence bands peaked at 480$500 \mathrm{~nm}$ appear. As can be seen, the observed spectra exhibit the isoemissive points whose location is dependent on the nature of an analyte.

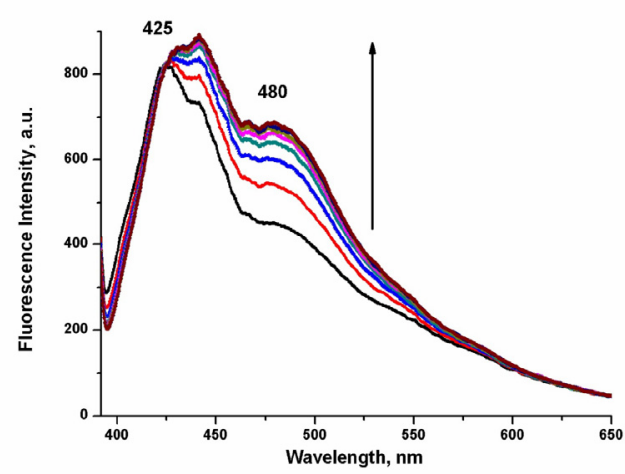

Fig. 3. Time-dependent fluorescence spectra of $D B M B F_{2}$-grafted SNP upon exposure to saturated benzene vapor (one measurement every $4 \mathrm{~s})$.

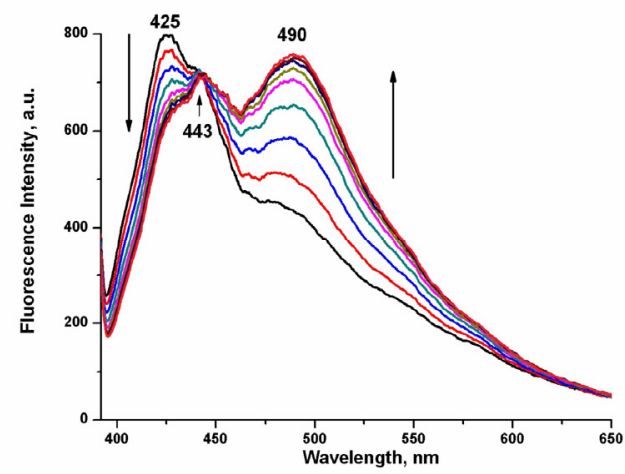

Fig. 4. Time-dependent fluorescence spectra of $D B M B F_{2}$-grafted SNP upon exposure to saturated toluene vapor (one measurement every 4 s).

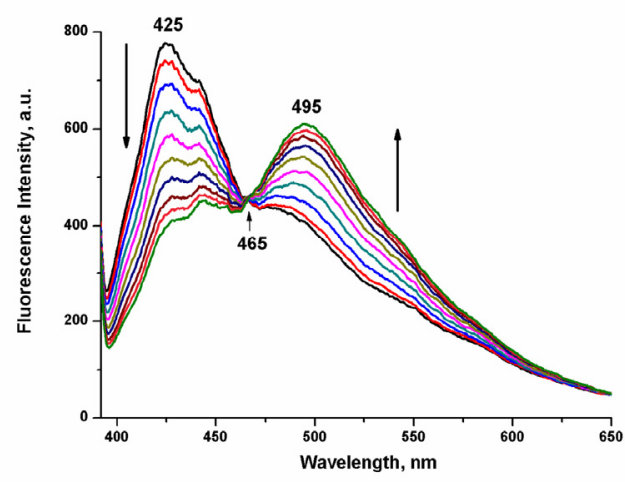

Fig. 5. Time-dependent fluorescence spectra of $D B M B F_{2}$-grafted SNP upon exposure to saturated $m$-xylene vapor (one measurement every $4 \mathrm{~s})$.

Such a behavior is analogous to that observed for $\mathrm{DBMBF}_{2}$ in solutions containing benzene or methyl-substituted benzenes [1-3] and must be related to formation of $\mathrm{DBMBF}_{2}$ /analyte exciplexes similar to exciplexes formed by 


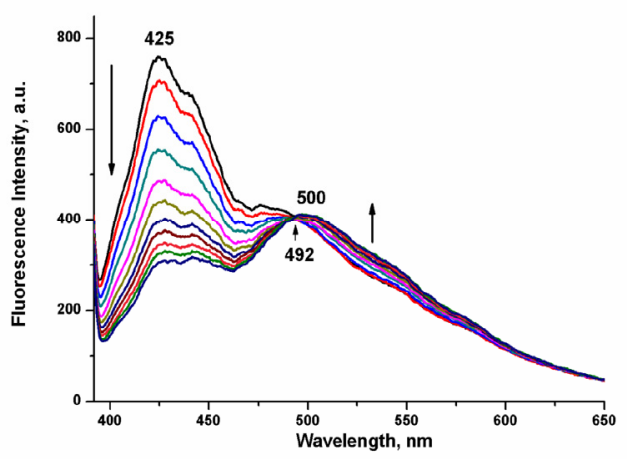

Fig. 6. Time-dependent fluorescence spectra of $D B M B F_{2}$-grafted SNP upon exposure to saturated $p$-xylene vapor (one measurement every $4 \mathrm{~s}$ ).

pyrene and dimethylaniline on the $\mathrm{SiO}_{2}$ surface [7]. Note that the positions of the exciplex fluorescence maxima and isoemissive points are considerable red-shifted in comparison with solutions. For example, the position of the isoemissive point of the $\mathrm{DBMBF}_{2} /$ toluene exciplex is comparable with the position of the isoemissive point of the $\mathrm{DBMBF}_{2} / 1,2,3,4-$ tetramethylbenzene exciplex in cyclohexane solution [1]. This may be a consequence of the presence of polar silanol groups in the vicinity of formed exciplexes.

It can be seen that the isoemissive point for benzene is located in the neighborhood of the $c$ fluorescence maximum ( $425 \mathrm{~nm})$, whereas for toluene the point is shifted to $443 \mathrm{~nm}$. Going from toluene to meta-xylene results in the shift to $465 \mathrm{~nm}$. It is worth noting that the spectral changes for ortho-xylene having the same ionization potential as meta-xylene are practically identical with those for meta-xylene (fluorescence maximum and isoemissive point are located at 497 and $467 \mathrm{~nm}$, respectively, as compared to 495 and $465 \mathrm{~nm}$ for meta-xylene). However, a further bathochromic shift to 492 $\mathrm{nm}$ is observed for para-xylene. Thus, the position of the isoemissive point differs markedly in the benzene-toluene-xylenes family and therefore it can be used for a decisive identification of an individual analyte.

From the results presented in Figs. 3-6 it is apparent that at a high concentration an analyte can be detected either by a decrease in the $\mathrm{DBMBF}_{2}$ fluorescence intensity or be an increase in the fluorescence intensity of corresponding exciplex. For the samples studied, the response times were equal to several tens of seconds. It is evident that the response time may depend on a number of factors, in particular, on the diffusion rate of analytes within a sensor layer and can be varied within wide limits by changing the composition and structure of the layer.

To test the possibility of detecting low concentrations of BTX, the fluorescence response to the presence of $12 \mathrm{ppm}$ orthoxylene was measured. The results are presented in Fig. 7.

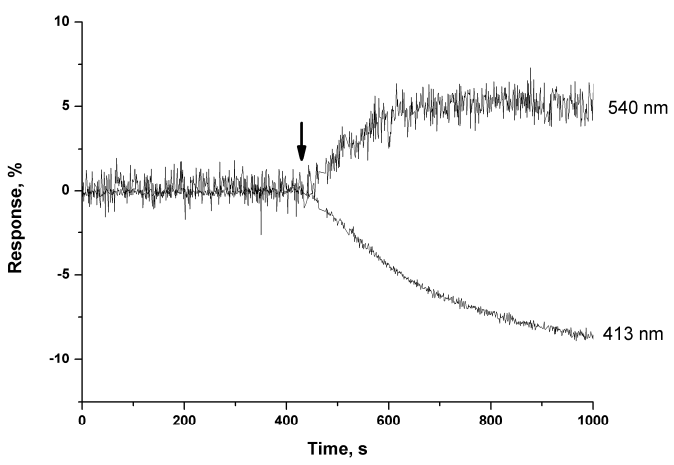

Fig. 7. Fluorescence response upon exposure to o-xylene vapor $(c=12 \mathrm{ppm})$ at two wavelengths. The arrow indicates the moment when o-xylene vapor was admitted.

As can be seen, the observed changes in the fluorescence intensity at both wavelengths corresponding to the fluorescence of $\mathrm{DBMBF}_{2}$ $(413 \mathrm{~nm})$ and its exciplex with o-xylene (540 $\mathrm{nm}$ ) are sufficient for a reliable registration of oxylene vapour even at such a low concentration.

The process of exciplex formation on the SNP surface may be suggested as follows. When a $\mathrm{DBMBF}_{2}$ molecule is electronically excited, its interaction with one of adsorbed analyte molecules leads to formation of a fluorescent $\mathrm{DBMBF}_{2} /$ analyte exciplex. It should be pointed out that an analyte molecule can form weak complex with a $\mathrm{DBMBF}_{2}$ molecule already in its ground electronic state. It may be inferred from the fact that the vapor exposure in the dark results in the same fluorescence response as exposure under continuous excitation.

\section{Conclusions}

Thus, core-shell silica nanoparticles with a shell consisting of covalently linked alkoxysilyl derivative of $\mathrm{DBMBF}_{2}$ were synthesized and investigated as sensitive and selective components of a sensor material suitable for detection of gaseous BTX. It was shown that the occurrence of the characteristic isoemissive points can be used for unique identification of a BTX analyte. This may offer new opportunities for qualitative and quantitative analysis of BTX mixtures. 


\section{Acknowledgements}

This work was supported by the Ministry of Education and Science of the Russian Federation (State Contract no. 02.527.11.0009 coordinated with the INGENIOUS project of the $7^{\text {th }}$ European Framework Program, grant no. 248236).

\section{References}

[1] Y.L. Chow, C.I. Johansson, Exciplexes of (Dibenzoylmethanato)boron/Benzenes: The Control of Exciplex Electronic Structure, The Journal of Physical Chemistry 99, 1755817565 (1995); doi:10.1021/j100049a015

[2] P. Valat, V. Wintgens, Y.L. Chow, and J. Kossanyi, Unusually strong emission from an exciplex formed between benzenoid solvents and dibenzoylmethanatoboron difluoride. Formation of a triplex, Canadian Journal of Chemistry 73, 1902-1913 (1995); doi:10.1139/v95-235

[3] T.-T. Truong, V. Brenner, G. Ledoux, T.-H. Tran-Thi, Exciplexes or ground state complexes of (dibenzoylmethanato)boron difluoride and benzene derivatives? A study of their optical properties revisited via liquid state investigations and structure calculations, Photochemical \& Photobiological Sciences 5, 686-697 (2006); doi:10.1039/b600710b
[4] V.A. Sazhnikov, V.M. Aristarkhov, A.G. Mirochnik, E.V. Fedorenko, M.V. Alfimov, Fluorescence Quenching of Silica GelAdsorbed (Dibenzoylmethanato)boron Difluoride by Polar Solvent Vapor,Doklady Physical Chemistry 437, 35-37 (2011); doi:10.1134/S0012501611030031

[5] V.A. Sazhnikov, V.M. Aristarkhov, A.A. Safonov, A.A. Bagatur'yants, A.G. Mirochnik, E.V. Fedorenko, M.V. Alfimov, Fluorescence Spectra and Structure of the Difluoro(dibenzoylmethanato)boron Monomers and Dimers Adsorbed on Silica Gel, High Energy Chemistry 45, 315-319 (2011); doi:10.1134/S0018143911030143

[6] A.A. Khlebunov, D.S. Ionov, P.V. Komarov, V.M. Aristarkhov, V.A. Sazhnikov, A.N. Petrov, M.V. Alfimov, An Experimental System for Investigating the Characteristics of Optical Sensor Materials, Instruments and Experimental Techiques 52, 132-136 (2009);

doi: $10.1134 / S 0020441209010229$

[7] G. Zhang, J.K. Thomas, A. Eremenko, T. Kikteva, F. Wilkinson, Photoinduced ChargeTransfer Reaction between Pyrene and N,N'-Dimethylaniline on Silica Gel Surfaces, The Journal of Physical Chemistry B, 101, 8569-8577 (1997); doi:10.1021/jp971983y 